\title{
Construcción de un Cuestionario de Habilidades Sociales para el Contexto Académico (CHS-A)
}

\section{Construction of a Social Skills Questionnaire for the Academic Context (CHS-A)}

\author{
Paula Salas ${ }^{1}$, Rodrigo Asún ${ }^{2}$ y Claudia Zúñiga ${ }^{3}$
}

\section{Resumen}

Se construye un cuestionario online de habilidades sociales para el contexto académico diseñado para estudiantes secundarios chilenos. Se realizaron dos estudios, uno con 583 participantes y otro con 3337. En el primero se construyó el cuestionario en base a la estructura propuesta por Del-Prette, Del-Prette y Mendes (1998) y se evaluó su fiabilidad y su validez basada en contenido, estructura y criterio externo. En el segundo estudio, se puso a prueba la estabilidad de la estructura resultante y se estimó la fiabilidad con la estructura final. Se obtuvo un instrumento con adecuadas propiedades psicométricas para usos descriptivos, con una estructura de tres factores: Asertividad, Conversación y Autorregulación. A la luz de estos resultados, se discute acerca de la dimensionalidad del constructo de habilidades sociales.

Palabras clave: habilidades sociales, cuestionario online, escala, validación, estudiantes secundarios

\begin{abstract}
An online questionnaire of social skills for an academic context was created for secondary public-school students. Two studies were carried out. In the first (583 participants) a questionnaire was constructed based on a structure proposed by Del-Prette, Del-Prette and Mendes (1998), and its reliability and validity based on content, structure and external criteria were assessed. In the second study, the stability of the resulting structure was tested and its reliability was estimated. It was obtained a questionnaire with adequate psychometric properties for descriptive uses, with a structure of three factors: Assertiveness, Conversation and Self-regulation. In the light of these results, the dimensionality of social skills is discussed.
\end{abstract}

Keywords: social skills, online questionnaire, scale, validation, secondary students

Esta investigación se ha realizado en el marco de los proyectos FONDEF IT15I10074 y CA13I10087 financiados por la Comisión Nacional de Investigación Científica y Tecnológica de Chile.

\footnotetext{
${ }^{1}$ Psicóloga. Licenciada en Psicología. Investigadora de la Universidad de Chile. Ignacio Carrera Pinto 1045, Ñuñoa, Santiago, Chile. Correo: paula.salas@ug.uchile.cl

${ }^{2}$ Sociólogo. Doctor en Psicología. Académico de la Universidad de Chile. Ignacio Carrera Pinto 1045, Nuñoa, Santiago, Chile. Correo: rasun@uchile.cl

${ }^{3}$ Psicóloga. Doctora en Psicología. Académica de la Universidad de Chile. Ignacio Carrera Pinto 1045, Nuñoa, Santiago, Chile. Correo: cczuniga@u.uchile.cl

Revista Iberoamericana de Diagnóstico y Evaluación - e Avaliação Psicológica. RIDEP · No55 · Vol.2 · 89-105 · 2020

ISSN: 1135-3848 print /2183-6051online
} 


\section{Introducción}

Las habilidades sociales (HS), entendidas como aquel conjunto de comportamientos necesarios para una adecuada interacción con otros, han demostrado ser de gran relevancia en el proceso de desarrollo social y personal (Bravo \& Herrera, 2011). Las relaciones interpersonales son nuestra principal fuente de bienestar, pero pueden también convertirse en la mayor causa de malestar (Roca, 2005), lo cual es especialmente cierto en el marco de las instituciones educativas (Bravo \& Herrera, 2011; Ramírez \& Justicia, 2006).

Numerosa evidencia muestra la existencia de una relación positiva entre habilidades sociales y logros escolares (Bravo \& Herrera, 2011). La falta de estas habilidades por su parte, se relaciona con diversas dificultades, como baja aceptación y aislamiento, problemas emocionales y escolares, desajustes psicológicos, entre otros (Bravo \& Herrera, 2011; Elliot \& Gresham, 1991; Hunder, 1995; Pelechano, 1996).

Respecto a la permanencia en el sistema educativo, las relaciones conflictivas con compañeros y profesores se encuentran entre las principales causas de la deserción escolar (UNESCO, 2012), y en el contexto de la educación superior, las HS influirían positivamente en el apoyo social percibido por los estudiantes y actuarían como un moderador frente al estrés, aumentando la capacidad de soportar las presiones académicas (Cohen, Sherrod, \& Clark, 1986; Riggio, Watring, \& Throckmorton, 1993; Villar, Caparrós, Romero, \& Camps, 2009).

El apoyo social percibido por los estudiantes incidiría en la retención universitaria (Robbins et al., 2004; Robbins, et al., 2006), lo que sería aún más importante en estudiantes de contextos socioeconómicos vulnerables, especialmente cuando son los primeros de su familia en ingresar a la educación superior, ya que muchas veces no cuentan con referentes de la vida universitaria (Canales \& De los Ríos, 2009).

Así, las HS tendrían un rol clave para la integración social en el contexto educativo y por consiguiente, en la permanencia de los estudiantes. De este modo, evaluar las HS de forma temprana, podría contribuir a prevenir la deserción escolar y preparar a los estudiantes para afrontar de mejor modo los desafíos de la transición a la educación superior.

Las HS han sido objeto de gran interés para distintas áreas de la psicología, tales como la psicología social, clínica y educativa (Eceiza, Arrieta, \& Goñi, 2008), pero a pesar del interés que despierta este constructo, ha resultado complejo definirlo y delimitar una taxonomía clara de lo que representan las HS (Caldarella \& Merrell, 1997; Lacunza \& Contini de González, 2011). Los principales problemas para llegar a un acuerdo en la definición del constructo han sido tres: la diversidad de términos con que se ha llamado al mismo concepto (conducta asertiva, asertividad, competencias sociales, entre otros); la dependencia de las HS del contexto en el que se manifiestan; y la diferencia entre las aproximaciones con énfasis en la conducta y las aproximaciones con énfasis en las consecuencias de la conducta (Betina Lacunza \& Contini de González, 2011; Cohen Imach, Esterkind de Chein, Betina Lacunza, Caballero, \& Martinenghi, 2010).

En cuanto a la estructura del constructo, ha habido aún más discordancia, proponiéndose una amplia diversidad de dimensiones. Caballo (1983) hace una revisión del concepto de asertividad, encontrando ocho taxonomías distintas planteadas entre 1973 y 1981, entre las cuales se proponen desde dos hasta ocho dimensiones. En la misma línea, Caldarella y Merrell (1997) hacen una revisión de 21 estudios en que se utilizan técnicas multivariadas para definir una taxonomía de HS en niños y adolescentes, encontrando que las estructuras propuestas van desde uno a cinco factores.

Sin embargo, a pesar de estas grandes divergencias, existe consenso al menos en dos puntos. El primero apunta a que este constructo, en un sentido general, se refiere a los tipos de conductas requeridas en las interacciones con otras personas, que permiten a un sujeto desenvolverse de un modo adecuado a la situación $\mathrm{y}$, generalmente, posibilitan la resolución de problemas (Caballo, 2005). Un segundo consenso apunta a la especificidad situacional de las HS, ya que las habilidades necesarias para desenvolverse adecuadamente en un contexto no son las mismas que las que se requieren en otra situación (Eceiza et al., 2008; Caballo 1983; Caldarella \& Merrell, 
1997). De este modo, se podría afirmar que las HS permiten desenvolverse y lograr objetivos interpersonales adecuándose a los requerimientos específicos de cada contexto social.

En este escenario de poca claridad acerca del constructo y gran interés sobre el mismo, se han desarrollado numerosos instrumentos de medición a lo largo de los años, tan diversos como los modelos teóricos a la base, para medir asertividad, dificultades sociales, ansiedad social, competencia social, entre otros constructos similares (Eceiza et al., 2008).

Entre los instrumentos más usados para medir habilidades sociales, dirigidos a población adolescente, se puede encontrar el Teenage Inventory of Social Skills ([TISS] Inderbitzen \& Foster, 1992; Inglés, Hidalgo, Méndez, \& Inderbitzen, 2003), la Escala Matson Evaluation of Social Skills with Youngsters ([MESSY] Matson, Rotatori, \& Helsel, 1983), la Escala Multidimensional de Expresión Social ([EMESM] Caballo, 1993), el Cuestionario de Dificultades Interpersonales ([DCI] Eceiza et al., 2008), el Inventario de Habilidades Sociales ([IHS] Del-Prette et al., 1998; Olaz, Medrano, Greco, \& Del-Prette, 2009), la Escala de Habilidades Sociales ([EHS]; Gismero, 2000; Miranda-Zapata et al, 2014), el Cuestionario de Evaluación de Dificultades Interpersonales en la Adolescencia ([CEDIA] Inglés, Méndez, \& Hidalgo, 2000), entre otros.

En concordancia con lo planteado por Eceiza et al. (2008), todos estos instrumentos evalúan las HS en términos globales, considerando los distintos contextos en el que ocurren las relaciones interpersonales (interacción con familia, pareja, entre otros), e incluyen escasos indicadores que puedan ser especialmente pertinentes para el contexto académico.

Por ello, el presente trabajo tuvo como objetivo construir un cuestionario para medir las habilidades sociales más requeridas en el contexto académico, dirigido a estudiantes secundarios chilenos. Se optó por crear y evaluar el funcionamiento del cuestionario con estudiantes provenientes de establecimientos educativos con mayor vulnerabilidad socioeconómica, ya que estos estos estudiantes suelen tener mayor temor en las relaciones sociales, timidez y sentimientos de aislamiento social (Coronel, Levin, \& Mejail, 2011).
Se decidió desarrollar el instrumento en formato online, pues resulta más atractivo para los estudiantes, se evitan errores de digitación y permite la generación de informes de devolución automatizados, facilitando la interpretación de los resultados.

Para la creación del nuevo cuestionario se tomó como punto de partida la estructura e ítems del IHS (Del-Prette et al., 1998). El IHS está compuesto de 38 afirmaciones tipo Likert, y si bien los autores no proponen una definición particular para las HS, las definen en base a una estructura que contempla cinco dimensiones:

1. Enfrentamiento del riesgo o asertividad: hace referencia a situaciones con riesgo potencial de una reacción indeseada por parte del interlocutor, en las que se requiere la afirmación de derechos y autoestima.

2. Expresión de afecto positivo: refiere a la expresión de afecto en situaciones con riesgo mínimo de una reacción indeseada.

3. Conversación y desenvoltura social: retratan situaciones sociales neutras que requieren manejo de las normas sociales cotidianas.

4. Auto-exposición a desconocidos o a situaciones nuevas: refiere al acercamiento e interacción con personas desconocidas.

5. Autocontrol de la agresividad en situaciones aversivas: refiere a situaciones en que es necesario controlar la rabia ante reacciones aversivas de parte del interlocutor.

El IHS ha demostrado adecuada fiabilidad en estudiantes universitarios tanto en Brasil (Alfa de Cronbach global $=.75$, con valores entre .74 y .96 por dimensión) (Del Prette \& Del Prette, 2013) como Argentina (Alfa de Cronbach global=.79, con valores entre .52 y .70 por dimensión) (Olaz et al., 2009), y presenta adecuadas evidencias de validez concurrente y estabilidad temporal en población brasileña (Bandeira, Costa, Del Prette, Del Prette, \& Gerk-Carneiro, 2000).

Se decidió trabajar en base a este cuestionario, ya que, a diferencia de otros instrumentos disponibles, su estructura factorial está conformada en función de las demandas que el sujeto debe enfrentar (e.g. expresar afectos, manejar la rabia), mientras que los distintos contextos en los que ocurre la interacción se distribuyen equitativamente entre dimensiones. Esto permite modificar el contexto de los ítems, 
adecuándolos a ámbitos relevantes para el contexto académico, sin modificar la estructura del instrumento. Lo mismo no ocurre con otros instrumentos que tienden a incluir dimensiones completas que se refieren a un solo contexto de interacción (e.g. como consumidor) y, por tanto, modificar el contexto de los ítems necesariamente implica modificar la estructura.

Se realizaron dos estudios consecutivos. En el primero se construyó el nuevo cuestionario usando como base la estructura e ítems del IHS y se evaluó su validez basada en contenido, estructura y criterio externo. Debido a que los resultados no fueron completamente satisfactorios, se llevó a cabo un segundo estudio en el que se puso a prueba la estabilidad de la nueva estructura y se estimó la fiabilidad de instrumento final.

\section{Estudio 1}

\section{Método}

\section{Instrumentos}

Cuestionario de habilidades sociales (CHS-A). Para la construcción del nuevo cuestionario se utilizaron como base los ítems del IHS, pero modificando el contenido para hacer referencia únicamente a situaciones que puedan ocurrir en el contexto académico o contextos inespecíficos. Esto implicó modificar ítems que hicieran referencia a, por ejemplo, interacciones con familia o como consumidor, pero manteniendo el tipo de demanda requerida en cada ítem. De esta forma, el nuevo instrumento se creó manteniendo la misma estructura planteada por Del Prette y Del Prette (1998).

Aunque existe una adaptación argentina del IHS, se decidió trabajar en base a la versión original en portugués para respetar el contenido y la estructura original. Los ítems fueron traducidos al castellano por uno de los miembros del equipo de investigación con manejo de ambos idiomas. Una vez traducidos fueron adaptados al contexto cultural y etario de la población objetivo por el equipo de investigación, que incluyó expertos en psicometría y en el constructo. Se seleccionaron los ítems pertinentes al ámbito académico, se modificaron o eliminaron los que no resultaran adecuados para ese contexto y se construyeron nuevos reactivos para reemplazar los eliminados. Así se obtuvo una primera versión del instrumento conformada por 35 ítems, manteniendo las cinco dimensiones originales.

Para aportar evidencias de validez basadas en el contenido de la prueba se recurrió al juicio de expertos. Se contó con cuatro jueces, expertos en la población objetivo, en el constructo y/o en psicometría. Se les presentó una pauta para evaluar tres elementos: (1) adecuación de las dimensiones para dar cuenta del constructo a medir, (2) pertinencia de las instrucciones y formato de respuesta para la población objetivo, y (3) pertinencia de los ítems para medir cada una de las dimensiones y su adecuación a la población objetivo.

Como resultado de este proceso se simplificó y adecuó la redacción de 13 ítems, se eliminó un ítem de la dimensión Expresión de afecto positivo y se construyeron cuatro nuevos ítems: dos para la dimensión de Autocontrol de la agresividad en situaciones aversivas y dos para la dimensión de Expresión de afecto positivo. Se decidió dejar todas las dimensiones con un mínimo de seis ítems, resultando un total de 38 reactivos. Paralelamente, se desarrolló la plataforma web que soporta el instrumento, usando el software LimeSurvey $^{1}$, que abastece automáticamente la base de datos con las respuestas y permite la generación automática de informes de resultados.

Se realizó una prueba piloto cualitativa con una muestra de cinco estudiantes con edades entre 17 y 19 años. El objetivo fue evaluar la claridad de las instrucciones y de los ítems, así como el funcionamiento de la plataforma online. Los estudiantes respondieron el instrumento y luego participaron de una entrevista grupal. No fue necesario realizar ningún cambio al instrumento tras esta etapa, ya que a los participantes les pareció comprensible y fácil de responder.

Una vez definida la primera versión del cuestionario se llevó a cabo una aplicación a gran escala con el fin de obtener sus propiedades psicométricas. Se aplicó la versión de 38 ítems del CHS-A, cuya construcción se ha descrito en los párrafos anteriores. Ante cada ítem el estudiante debía señalar la frecuencia con que

\footnotetext{
${ }^{1}$ Aplicación web open source para la aplicación de encuestas en línea.
} 
actúa de la forma descrita, escogiendo entre cinco categorías de respuesta (Nunca, Casi nunca, A veces, Casi siempre y Siempre) puntuadas de 1 a 5 en el caso de 23 ítems que están redactados de forma directa (i.e. mayor frecuencia indica mayor nivel de la variable) y puntuadas de forma inversa en el caso de 15 ítems formulados de modo que mayor frecuencia refleja menor nivel de la variable.

Escala de Detección de la Ansiedad Social (EDAS). Además del CHS-A, se aplicó la EDAS con el fin de aportar evidencias de validez convergente del nuevo instrumento. Esta escala está formada por tres subescalas (evitación, ansiedad e interferencia) y 26 ítems. Los dos primeros son dicotómicos (Sí/No) y no son considerados en la puntuación total. Los 24 restantes son tipo Likert, con cinco alternativas de respuesta y describen situaciones sociales difíciles para personas con ansiedad social. Se seleccionó la EDAS como instrumento para analizar la validez convergente del CHS-A debido a que al momento de la aplicación era uno de los pocos instrumentos para evaluar variables relacionadas con HS que contaban con evidencia de adecuadas propiedades psicométricas en población adolescente chilena (Piqueras et al, 2012, Vera-Villarroel et al., 2007;). La EDAS ha presentado adecuadas propiedades psicométricas, con alfas de Cronbach de $.80, .84 \mathrm{y}$ .86 para las subescalas de evitación, ansiedad e interferencia respectivamente y adecuados indicadores de ajuste de la estructura unifactorial de cada subescala, con un índice de ajuste GFI mayor a .99 y una media de los residuales menor a -.0002 (Vera-Villarroel et al., 2007).

\section{Participantes}

La población objetivo fueron estudiantes de enseñanza secundaria de colegios públicos, de dos regiones chilenas. Los colegios fueron seleccionados a través de un muestreo probabilístico por conglomerados polietápico, donde la primera selección correspondió al establecimiento y la segunda al curso. Se invitó a participar a todos los estudiantes de los cursos seleccionados, resultando en un promedio de participación de 41.64 estudiantes por colegio ( $D T=46.65)$. Así, la muestra quedó conformada por 583 estudiantes entre 13 y 19 años $(M=15.78$ años, $D T=1.15$ ) de 14 colegios. El 51.8\% fueron mujeres y $48.2 \%$ hombres.
No todos los participantes respondieron la EDAS, ya que este estudio se enmarcó en un proyecto de investigación más amplio que incluía otros instrumentos de medición. Con el fin de evitar el agotamiento de los estudiantes, se seleccionó de forma aleatoria una submuestra de 304 estudiantes, a quienes se aplicó el EDAS.

\section{Procedimiento}

Se solicitó autorización a las autoridades de cada colegio seleccionado, para aplicar el cuestionario en horario de clases. Una vez obtenida dicha autorización, se envió a los apoderados un formulario de consentimiento informado que explicitaba los objetivos e implicancias de la investigación. A los estudiantes que contaban con el consentimiento de sus apoderados, se les solicitó además su asentimiento.

La aplicación se realizó en forma colectiva en la sala de informática del colegio o en una sala de clases, con computadores portátiles. Las instrucciones fueron entregadas por un investigador, quien además respondió las consultas de los estudiantes y tomó nota de ellas. No se identificaron problemas de comprensión de los ítems o las instrucciones.

\section{Análisis de Datos}

\section{Análisis clásico de ítems}

Para evaluar la distribución de las respuestas de los sujetos ante cada reactivo, se calculó la media, desviación típica, asimetría y curtosis de cada ítem. Además, para cada dimensión, se evaluó la discriminación de los ítems a través de correlaciones ítem-test poliseriales corregidas. Para estos análisis se utilizaron los software IBMSPSS-22 y Mplus 7.4.

\section{Evidencias de validez basadas en estructura interna}

Utilizando el programa Mplus-7.4 se realizaron análisis factoriales exploratorios (AFE) y confirmatorios (AFC) utilizando el método de mínimos cuadrados ponderados robustos (WLSMV). Se trabajó en base a la matriz de correlaciones policóricas, considerando la naturaleza ordinal de las respuestas (YangWallentin, Jöreskog, \& Luo, 2010). 
Para evaluar la bondad de ajuste global de los modelos se utilizó el índice Chi cuadrado. Sin embargo, debido a la sensibilidad de este estadístico al tamaño muestral, se complementó con una medida del índice Chi cuadrado dividido en los grados de libertad $\left(\chi^{2} / g l\right)$, para el cual valores cercanos o menores a 3 se consideraron adecuados (Martínez, Hernández, \& Hernández, 2006). Adicionalmente se analizaron tres de los índices de ajuste más utilizados y con menor dependencia del tamaño muestral según Abad, Olea, Ponsoda y García (2011). Estos son: el índice de bondad de ajuste absoluto RMSEA, considerando adecuados valores menores a .08 (Martínez et al., 2006) y los índices de bondad de ajuste incremental TLI y CFI, en que valores mayores a .9 se consideraron aceptables (Ferrando \& Anguiano-Carrasco, 2010). Además, se consideraron adecuados ítems con cargas factoriales (lambda) mayores a .4 (Ferrando \& Anguiano-Carrasco, 2010).

\section{Análisis de la consistencia interna}

Se estimó a través del estadístico Alfa Ordinal, sugerido para escalas tipo Likert (Elosua \& Zumbo, 2008).

\section{Análisis descriptivo}

Se calcularon media, cuartiles, desviación típica y coeficiente de asimetría para cada dimensión y para la escala total. Además, se determinó la asociación con la variable sexo.

\section{Evidencias de validez convergente}

Para la submuestra que respondió la EDAS, se calculó el coeficiente de correlación Rho de Spearman entre cada una de las dimensiones del CHS-A y el puntaje total de la EDAS.

\section{Resultados}

\section{Análisis clásico de ítems}

En la Tabla 1 se observa una tendencia hacia la asimetría negativa, acumulándose las mayores frecuencias de respuesta en los valores altos de la escala. Sin embargo, no se observan desviaciones típicas demasiado bajas, lo que indica que incluso los ítems con mayor asimetría tienen variabilidad en las respuestas.
Todos los ítems presentan correlaciones significativas con la puntuación total de la dimensión. Asimismo, la mayor parte de los ítems presentan correlaciones moderadas con su respectiva dimensión, lo que indica que esos ítems logran diferenciar a los sujetos con altos y bajos niveles de habilidad en cada dimensión. Sin embargo, también se observan algunos ítems con una baja capacidad discriminadora entre altas y bajas puntuaciones, y por tanto su funcionamiento fue revisado en subsecuentes análisis.

\section{Evidencias de validez basadas en estructura interna}

Considerando el poco acuerdo existente en torno a la estructura del constructo de habilidades sociales, y que se realizaron cambios profundos al instrumento original IHS, resultando un cuestionario muy diferente, se decidió realizar en primer lugar un análisis factorial exploratorio (AFE). Se utilizó el método WLSMV considerando la naturaleza ordinal de las respuestas, y una rotación oblicua para permitir la correlación entre dimensiones. Esto arrojó una solución de cuatro factores con adecuado ajuste $\left(\chi^{2}=218.58 ; \chi^{2} / \mathrm{gl}=2.19 ;\right.$ RMSEA $=.045 ;$ CFI $=.91 ;$ TLI=.89). Sin embargo, el cuarto factor incluía solo tres ítems (ninguno con un alto lambda) y al evaluar teóricamente su contenido, este no resultó sustantivo, por lo que se consideró un factor residual y se optó por eliminarlo.

En base a estos resultados, se propuso una nueva estructura de tres factores que se evaluó mediante una serie de AFC en los cuales se fueron eliminando ítems con bajas cargas factoriales (menores a .4) y/o inadecuados teóricamente para medir dichos factores, obteniéndose finalmente un modelo con un adecuado ajuste $\left(\chi^{2}=362.79\right.$; $\chi^{2} / \mathrm{gl}=3.13$; RMSEA=.06; CFI=.91; TLI=.90) у teóricamente coherente. Así, la nueva versión del instrumento se compone por 17 ítems y tres factores, tal como se aprecia en la Figura 1.

En esta nueva estructura, los ítems de la dimensión Auto-exposición a desconocidos o a situaciones nuevas se agrupan con los de Conversación y desenvoltura social, conformado una dimensión que hemos denominado Conversación, mientras que dos de los ítems que se habían propuesto para esta dimensión se agrupan con los de Asertividad ("Puedo hacer 
Tabla 1. Análisis clásico de ítems Estudio 1

\begin{tabular}{|c|c|c|c|c|c|}
\hline Ítem & M & DT & Asimetría & Curtosis & $\begin{array}{l}\text { Correlación ítem- } \\
\text { dimensión }\end{array}$ \\
\hline \multicolumn{6}{|c|}{ Conversación y desenvoltura social } \\
\hline Ítem 1 & 2.83 & 1.134 & .163 & -.590 & $.419^{*}$ \\
\hline Ítem 8 & 3.71 & 1.196 & -.599 & -.606 & $.397^{*}$ \\
\hline Ítem 11 & 3.09 & 1.322 & -.063 & -1.082 & $.346^{*}$ \\
\hline Ítem 14 & 3.19 & 1.321 & -.229 & -1.027 & $.408^{*}$ \\
\hline Ítem 17 & 3.46 & 1.182 & -.363 & -.744 & $.556^{*}$ \\
\hline Ítem 20 & 3.30 & 1.206 & -.243 & -.823 & $.406^{*}$ \\
\hline Ítem 22 & 3.49 & 1.325 & -.431 & -.997 & $.191^{*}$ \\
\hline Ítem 30 & 3.53 & 1.050 & -.308 & -.402 & $.434^{*}$ \\
\hline Ítem 33 & 2.77 & 1.310 & .279 & -.979 & $.388^{*}$ \\
\hline Ítem 37 & 4.09 & .902 & -.600 & -.574 & $.328 *$ \\
\hline \multicolumn{6}{|c|}{ Enfrentamiento del riesgo } \\
\hline Ítem 3 & 3.52 & 1.138 & -.327 & -.660 & $.466^{*}$ \\
\hline Ítem 5 & 3.24 & 1.419 & -.201 & -1.238 & $.246^{*}$ \\
\hline Ítem 10 & 3.58 & 1.258 & -.515 & -.813 & $.525^{*}$ \\
\hline Ítem 15 & 3.92 & 1.115 & -.811 & -.119 & $.526^{*}$ \\
\hline Ítem 19 & 4.50 & .908 & -1.860 & 2.855 & $.371^{*}$ \\
\hline Ítem 24 & 3.38 & 1.254 & -.350 & -.820 & $.291^{*}$ \\
\hline Ítem 26 & 3.91 & 1.012 & -.735 & .007 & $.529^{*}$ \\
\hline Ítem 29 & 3.67 & 1.163 & -.493 & -.642 & $.376^{*}$ \\
\hline Ítem 31 & 3.82 & .989 & -.529 & -.188 & $.427^{*}$ \\
\hline Ítem 34 & 3.66 & 1.110 & -.533 & -.399 & $.193^{*}$ \\
\hline \multicolumn{6}{|c|}{ Expresión de afecto positivo } \\
\hline Ítem 2 & 3.75 & 1.123 & -.729 & -.062 & $.293^{*}$ \\
\hline Ítem 6 & 4.64 & .725 & -2.373 & 6.064 & $.448^{*}$ \\
\hline Ítem 13 & 3.40 & 1.316 & -.332 & -1.018 & $.200^{*}$ \\
\hline Ítem 18 & 4.03 & 1.014 & -.904 & .261 & $.450^{*}$ \\
\hline Ítem 27 & 3.97 & 1.056 & -.754 & -.279 & $.444^{*}$ \\
\hline Ítem 35 & 4.09 & .969 & -.879 & .262 & $.494^{*}$ \\
\hline \multicolumn{6}{|c|}{ Auto-exposición a desconocidos o a situaciones nuevas } \\
\hline Ítem 4 & 3.34 & 1.212 & -.360 & -.727 & $.403 *$ \\
\hline Ítem 7 & 3.17 & 1.233 & -.044 & -.930 & $.320^{*}$ \\
\hline Ítem 21 & 3.24 & 1.202 & -.132 & -.856 & $.455^{*}$ \\
\hline Ítem 25 & 3.25 & 1.333 & -.219 & -1.055 & $.313^{*}$ \\
\hline Ítem 32 & 3.78 & 1.227 & -.723 & -.492 & $.371^{*}$ \\
\hline Ítem 36 & 3.05 & 1.114 & -.069 & -.586 & $.541^{*}$ \\
\hline \multicolumn{6}{|c|}{ Autocontrol de la agresividad en situaciones aversivas } \\
\hline Ítem 9 & 4.10 & 1.049 & -1.106 & .640 & $.388^{*}$ \\
\hline Ítem 12 & 2.75 & 1.242 & .131 & -1.008 & $.350^{*}$ \\
\hline Ítem 16 & 3.42 & 1.007 & -.470 & .006 & $.548^{*}$ \\
\hline Ítem 23 & 3.95 & .983 & -.735 & .025 & $.423^{*}$ \\
\hline Ítem 28 & 4.18 & 1.061 & -1.324 & 1.113 & $.445^{*}$ \\
\hline Ítem 38 & 3.89 & 1.006 & -.719 & .097 & $.370^{*}$ \\
\hline
\end{tabular}

Nota. $M=$ media; $D T=$ desviación típica. Rango de respuestas de 1 a $5 ;$ * $p<.05$. Correlación ítem-dimensión corresponde a la correlación poliserial corregida. 


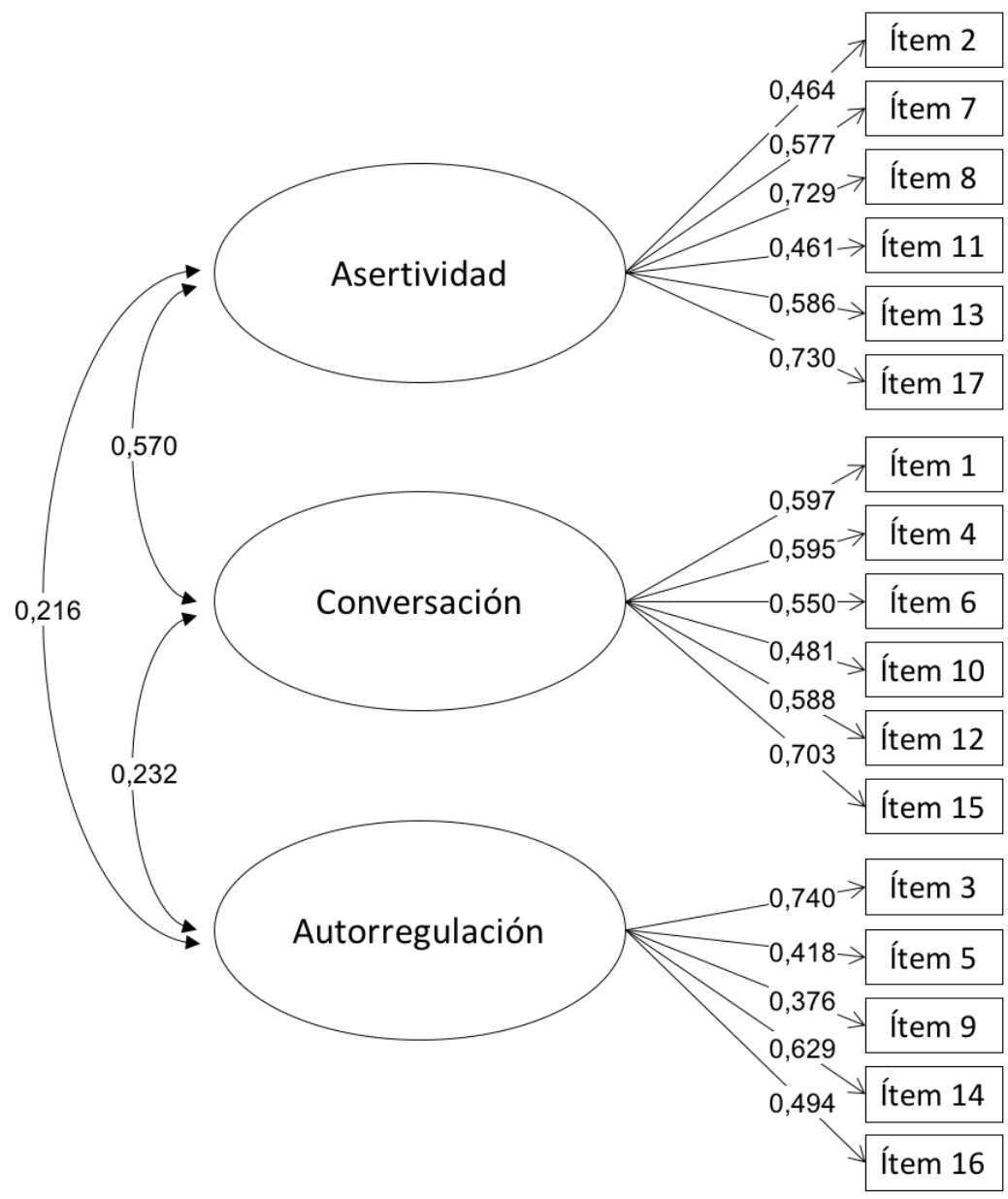

\begin{tabular}{|l|l|}
\hline$\chi^{2}$ & 362.791 \\
\hline gl & 116 \\
\hline$\chi^{2} / \mathrm{gl}$ & 3.13 \\
\hline RMSEA & .060 \\
\hline CFI & .91 \\
\hline TLI & .90 \\
\hline
\end{tabular}

Figura 1. Modelo de 3 factores y 17 ítems

disertaciones frente a mis compañeros sin ponerme nervioso" y "Si me interesa un tema de la clase, me acerco a conversar con el profesor"), posiblemente debido a que su contenido describe conductas con un mayor nivel de riesgo para los estudiantes. Los ítems de las dimensiones Expresión de afecto positivo y Autocontrol de la agresividad en situaciones aversivas se agrupan en una sola dimensión, que se ha denominado Autorregulación. En la Tabla 2 se presentan los ítems correspondientes a cada dimensión en la nueva estructura propuesta.

\section{Análisis de la consistencia interna}

Se obtuvieron coeficientes de .761 , y .757 para las escalas de Asertividad y Conversación, respectivamente, valores que se consideran adecuados para mediciones con fines descriptivos (Prieto \& Delgado, 2010). La dimensión de Autorregulación se encuentra en el límite, con un coeficiente de .658 , mientras que el cuestionario en su totalidad presenta un alfa ordinal de .947 .

\section{Análisis descriptivo}

Se observa una asimetría negativa en las tres dimensiones, aunque especialmente en Autorregulación, tal como muestra la Tabla 3. Esto indica que la mayor parte de los participantes presenta altas puntuaciones en las distintas dimensiones y en la escala total.

Al realizar comparaciones por género, se encuentran diferencias significativas pero pequeñas, entre hombres y mujeres en Asertividad $\left(t_{(577.3)}=-3.745 ; p<.001 ; d=-.31 ; 95 \%\right.$ IC[-2.15, .671]) y Autorregulación $\left(t_{(581)}=2.638 ; p=.009\right.$; $d=.22 ; 95 \%$ IC[.175, 1.192]), siendo los hombres quienes obtienen mayores puntuaciones en Asertividad y las mujeres en Autorregulación. En la dimensión Conversación y en la puntuación total del cuestionario, no se observan diferencias significativas (Conversación: $t_{(581)}=.91 ; p=.363$; $d=.08 ; 95 \%$ IC[-.398, 1.086]; Total: $t_{(581)}=-.528$; $p=.598 ; \quad d=-.04 ; \quad 95 \% \quad$ IC $[-1.807, \quad 1.041])$. 
Tabla 2. Estructura de la versión de 3 factores y 17 ítems

\begin{tabular}{|c|c|}
\hline \multicolumn{2}{|c|}{ Asertividad } \\
\hline İtem 2 & Si alguien me interrumpe cuando estoy hablando, soy capaz de pedirle que me deje terminar. \\
\hline Ítem 7 & Puedo hacer disertaciones frente a mis compañeros sin ponerme nervioso. \\
\hline Ítem 8 & Si en un grupo de personas conocidas estoy en desacuerdo con la mayoría, puedo expresar mi opinión. \\
\hline Ítem 11 & Si me interesa un tema de la clase, me acerco a conversar con el profesor. \\
\hline Ítem 13 & Si un amigo hace algo que me molesta, se lo hago saber. \\
\hline Ítem 17 & Si un profesor dice algo con lo que no estoy de acuerdo, me atrevo a darle mi opinión. \\
\hline \multicolumn{2}{|r|}{ 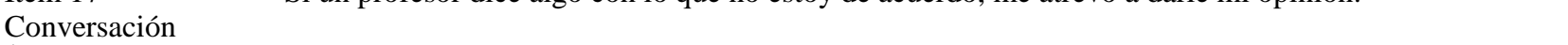 } \\
\hline Ítem 1 & En un grupo de personas desconocidas, me siento cómodo y puedo conversar normalmente. \\
\hline Ítem 4 & Aunque esté con personas conocidas, me cuesta integrarme a las conversaciones. \\
\hline Ítem 6 & Me cuesta terminar las conversaciones y en general me quedo esperando a que otros lo hagan. \\
\hline Ítem 10 & Evito acercarme a hablar con personas que parecen muy distintas a mí. \\
\hline Ítem 12 & Aunque necesite alguna información evito preguntar a desconocidos. \\
\hline Ítem 15 & Cuando estoy con una persona que acabo de conocer, me cuesta mantener una conversación. \\
\hline \multicolumn{2}{|r|}{ 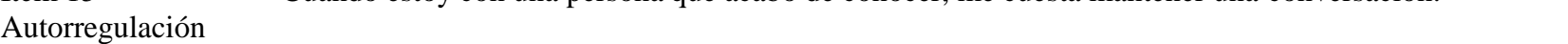 } \\
\hline Ítem 3 & Si hice sentir mal a un compañero le pido disculpas. \\
\hline Ítem 5 & Si un profesor me llama la atención, reacciono de forma respetuosa. \\
\hline Ítem 9 & Si un compañero me critica por algo, reacciono mal. \\
\hline Ítem 14 & Cuando un compañero se siente mal, trato de apoyarlo. \\
\hline Ítem 16 & Logro tomar como bromas las "tallas" que me hacen mis compañeros y no enojarme por ellas. \\
\hline
\end{tabular}

Tabla 3. Estadísticos descriptivos

\begin{tabular}{lccccccc}
\hline Dimensión & Rango posible & M & DT & Mdn & Q1 & Q3 & Asimetría \\
\hline Estudio 1 & & & & & & & \\
Asertividad & $6-30$ & 21.22 & 4.62 & 21 & 18 & 25 & -.331 \\
Conversación & $6-30$ & 19.47 & 4.56 & 19 & 17 & 23 & -.135 \\
Autorregulación & $5-25$ & 19.53 & 3.14 & 20 & 18 & 22 & -.695 \\
Escala total & $17-85$ & 60.21 & 8.75 & 60 & 54 & 66 & -.32 \\
Estudio 2 & & & & & & & \\
Asertividad & $6-30$ & 21.92 & 4.26 & 22 & 19 & 25 & -.38 \\
Conversación & $5-25$ & 17.19 & 3.68 & 17 & 15 & 20 & -.22 \\
Autorregulación & $4-20$ & 16.56 & 2.50 & 17 & 15 & 18 & -.76 \\
Escala total & $15-75$ & 55.68 & 7.61 & 56 & 50 & 61 & -.16 \\
\hline
\end{tabular}

Nota. $M=$ media; $D T=$ desviación típica; $M d n=$ mediana; $Q=$ cuartil.

$t_{(581)}=-.528 ; \quad p=.598 ; \quad d=-.04 ; \quad 95 \% \quad \mathrm{IC}[-1.807$, $1.041])$.

\section{Evidencias de validez convergente}

Antes de analizar la correlación entre la nueva versión del CHS-A y la EDAS, se evaluaron las propiedades psicométricas de la EDAS. Pese a tratarse de una escala validada en estudios previos, se observó un ajuste óptimo sólo en algunos de los índices que estimamos para cada dimensión (Evitación: $\quad \chi^{2}=78.400 ; \quad \chi^{2} / \mathrm{gl}=3.92$; RMSEA $=.098 ;$ CFI=.976; TLI=.967; Ansiedad: $\chi^{2}=65.959 ; \quad \chi^{2} / \mathrm{gl}=3.30 ; \quad \mathrm{RMSEA}=.087 ; \quad \mathrm{CFI}=.98 ;$ TLI=.972; Interferencia: $\chi^{2}=87.228 ; \chi^{2} / \mathrm{gl}=4.36$; RMSEA $=.105 ; \quad$ CFI $=.975 ; \quad$ TLI=.965), aunque presentó alfas ordinales adecuados (Evitación=.90; Ansiedad=.90; Interferencia=.91). Dado que no era objetivo de esta investigación modificar el EDAS, sino sólo usarlo como instrumento de validación convergente, decidimos continuar con esa tarea, pues la adecuada fiabilidad de las dimensiones del EDAS nos indica que se puede confiar gruesamente en las puntuaciones sujeto obtenidas con él, pese a que posiblemente se podría intervenir algunos de sus ítems para mejorar la validez de su estructura interna.

Por lo anterior, se estimó la correlación entre el CHS-A y la EDAS, obteniendo correlaciones inversas y significativas para cada una de las dimensiones y para la escala total (Asertividad: $r=-$ $.522 ; \quad p<.001 ;$ Conversación: $r=-.659 ; \quad p<.001$; Autorregulación: $r=-.169 ; p<.001$; Total: $r=-.677$; $p<.001$ ), aportando evidencia de que ambos instrumentos miden constructos relacionados, pero opuestos.

La correlación significativa pero baja entre la EDAS y la dimensión Autorregulación del CHSA es esperable, ya que la EDAS no incluye expresamente la regulación y expresión de afectos, como si lo hace esta dimensión. 


\section{Conclusiones}

Se obtuvo un instrumento de 17 ítems con adecuada fiabilidad y que cuenta con evidencias de validez convergente. Aunque la estructura del instrumento sufrió importantes modificaciones y no se mantuvieron las mismas dimensiones, la nueva versión mantuvo hasta cierto punto la coherencia teórica de la propuesta inicial, agrupando ítems y dimensiones teóricamente similares.

Sin embargo, debido a que la estructura final fue definida mediante AFE y AFC realizados con la misma muestra, se decidió realizar un segundo estudio con una muestra distinta, más heterogénea, pero del mismo universo de estudiantes secundarios, con el objetivo de evaluar la estabilidad de la nueva estructura propuesta para el CHS-A.

\section{Estudio 2}

\section{Método}

\section{Instrumentos}

Se aplicó el CHS-A en su versión de 17 ítems y tres dimensiones, desarrollada en el primer estudio.

\section{Participantes}

Se utilizó un muestreo no probabilístico por conveniencia, ya que los participantes fueron los estudiantes del Programa de Acompañamiento y Acceso Efectivo a la Educación Superior (PACE), del Ministerio de Educación de Chile. La muestra estuvo compuesta por 3870 estudiantes de 64 colegios de seis regiones del país. Se eliminaron aquellos casos que respondieron en cadena (misma categoría de respuesta a todos los ítems) o de forma inconsistente, quedando un total de 3337 estudiantes entre 16 a 22 años, con una media de 17.7 años $(D T=.775)$. El $54.4 \%$ fueron mujeres y $45.6 \%$ hombres.

\section{Procedimiento}

La aplicación fue coordinada con el programa PACE y los colegios participantes. El procedimiento fue el mismo que en el estudio 1.

\section{Análisis de datos}

Se realizaron los mismos análisis que en el estudio 1, excepto la validación convergente. Para evaluar el ajuste de la estructura factorial se utilizó solo AFC, considerando los mismos indicadores de ajuste que los usados previamente.

\section{Resultados}

\section{Análisis clásico de ítems}

Al igual que en el primer estudio, los ítems mostraron una tendencia hacia las puntuaciones altas, con medias en torno a los tres y cuatro puntos, asimetrías negativas, pero sin desviaciones demasiado bajas (ver Tabla 4). En cuanto a la discriminación, las correlaciones ítemdimensión corregidas resultaron significativas y moderadas, lo cual indica que los ítems logran discriminar entre sujetos con altos y bajos niveles en cada dimensión.

\section{Análisis de la estructura del instrumento}

Se analizó el ajuste a los datos de la estructura tridimensional propuesta, encontrándose índices de ajuste no adecuados $\left(\chi^{2}=2429.264 ; \chi^{2} / \mathrm{gl}=20.94\right.$; RMSEA=.077; CFI=.87; TLI=.85). Se revisaron las cargas factoriales y los índices de modificación entregados por el programa, con lo que se decidió eliminar dos ítems pertenecientes a los factores Conversación y Autorregulación, ya que uno presentó una baja carga factorial (ítem 9), y el otro presentó alta asociación con dos factores a la vez (ítem 1). Con estos cambios, el modelo mostró mejoras en los índices de ajuste $\left(\chi^{2}=1194.307 ; \quad \chi^{2} / \mathrm{gl}=13.73 ; \quad\right.$ RMSEA $=.062 ;$ $\mathrm{CFI}=.93$; TLI=.91) manteniendo la estructura de tres dimensiones (ver Figura 2). Si bien el índice $\chi^{2} / \mathrm{gl}$ aún presenta un valor alto, dado que muestras grandes también afectan a este índice (Newsom, 2005) y el resto de índices de ajuste tienen valores adecuados se consideró aceptable esta estructura.

\section{Análisis de la consistencia interna}

El coeficiente alfa ordinal resultó adecuado para fines descriptivos, exceptuando la dimensión de Autorregulación, que se encuentra en el límite (Asertividad $=.770$,

Conversación $=.712$, Autorregulación $=.635$, Total $=.884$ ). 
Tabla 4. Análisis clásico de ítems Estudio 2

\begin{tabular}{|c|c|c|c|c|c|}
\hline Ítem & M & DT & Asimetría & Curtosis & $\begin{array}{c}\text { Correlación ítem- } \\
\text { dimensión }\end{array}$ \\
\hline \multicolumn{6}{|c|}{$\overline{\text { Asertividad }}$} \\
\hline Ítem 2 & 3.53 & 1.079 & -.334 & -.524 & $.460 *$ \\
\hline Ítem 7 & 3.47 & 1.213 & -.443 & -.670 & $.450 *$ \\
\hline Ítem 8 & 3.95 & 1.020 & -.694 & -.233 & $.588 *$ \\
\hline Ítem 11 & 3.25 & 1.181 & -.183 & -.748 & $.404 *$ \\
\hline Ítem 13 & 3.96 & 1.010 & -.777 & .088 & $.480 *$ \\
\hline Ítem 17 & 3.66 & 1.147 & -.493 & -.562 & $.626 *$ \\
\hline \multicolumn{6}{|c|}{ Conversación } \\
\hline Ítem 1 & 3.12 & 1.076 & -.090 & -.415 & $.303 *$ \\
\hline Ítem 4 & 3.66 & 1.162 & -.542 & -.568 & $.493 *$ \\
\hline Ítem 6 & 3.47 & 1.144 & -.330 & -.662 & $.425 *$ \\
\hline Ítem 10 & 3.44 & 1.168 & -.316 & -.718 & $.411 *$ \\
\hline Ítem 12 & 3.37 & 1.123 & -.256 & -.637 & $.441 *$ \\
\hline Ítem 15 & 3.09 & 1.110 & -.056 & -.577 & $.531 *$ \\
\hline \multicolumn{6}{|c|}{ Autorregulación } \\
\hline Ítem 3 & 4.08 & 1.006 & -.963 & .373 & $.490 *$ \\
\hline Ítem 5 & 4.22 & .999 & -1.300 & 1.263 & $.369^{*}$ \\
\hline Ítem 9 & 3.6 & 1.005 & -.444 & -.140 & $.316^{*}$ \\
\hline Ítem 14 & 4.26 & .902 & -1.133 & .840 & $.420 *$ \\
\hline Ítem 16 & 3.89 & 1.005 & -.638 & -.143 & $.353 *$ \\
\hline
\end{tabular}

Nota. $M=$ media; $D T=$ desviación típica. Rango de respuestas de 1 a $5 ; * p<.05$. Correlación ítem-dimensión corresponde a la correlación poliserial corregida.

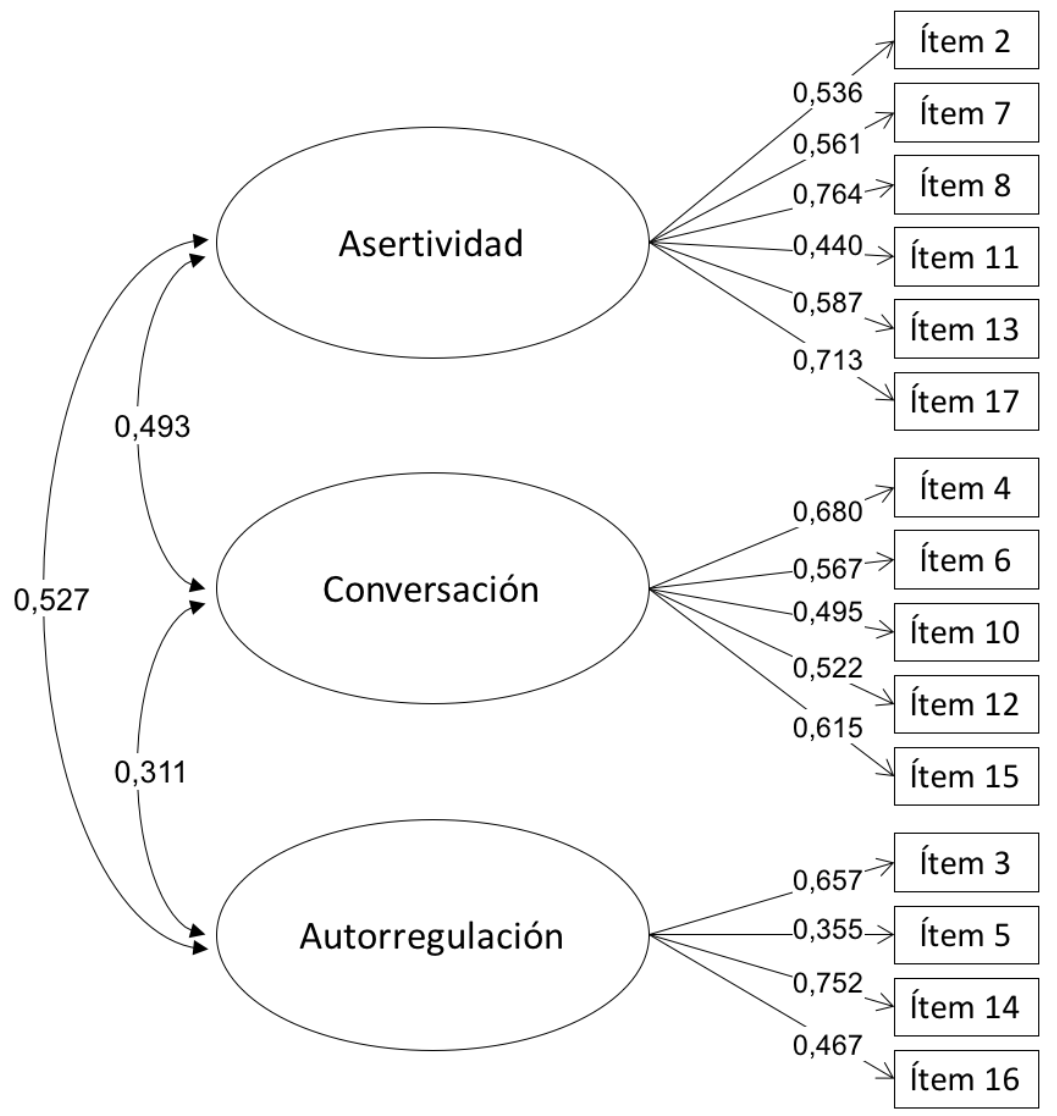

\begin{tabular}{|c|c|}
\hline$\chi^{2}$ & 1194.307 \\
\hline $\mathrm{gl}$ & 87 \\
\hline$\chi^{2} / \mathrm{gl}$ & 13.73 \\
\hline RMSEA & .062 \\
\hline CFI & .93 \\
\hline TLI & .91 \\
\hline
\end{tabular}

Figura 2. Modelo de 3 factores y 15 ítems

\section{Análisis descriptivo}

Se observó una asimetría negativa en las tres dimensiones y en la puntuación total, tal como se muestra en la Tabla 2, manteniéndose la tendencia hacia las altas puntuaciones observada en el primer estudio. También se replicó la asimetría levemente mayor en la dimensión Autorregulación. Al comparar por género, se replican las diferencias significativas y pequeñas del estudio 1 a favor de los hombres en Asertividad $\left(t_{(3277.3)}=-2.451 ; p=.014\right.$; $d=.08 ; 95 \%$ IC[-.650, -.072]) y a favor de las 
mujeres en Autorregulación $\left(t_{(3326)}=4.227\right.$; $p<.001 ; d=.15 ; 95 \%$ IC $[.197, .537])$. Pero además se encuentran diferencias significativas a favor de las mujeres en Conversación $\left(t_{(3326)}=3.215\right.$; $p=.001 ; d=.11 ; 95 \% \quad$ IC $[.128, .160])$. En la puntuación total estas diferencias no son significativas $\left(t_{(3326)}=1.572 ; p=.116 ; d=.05 ; 95 \%\right.$ IC[-.103, .936]).

\section{Conclusiones}

Se confirma una estructura de tres dimensiones para el CHS-A, similar a la propuesta en el estudio 1, pero obteniéndose una versión más breve, que con tan solo 15 ítems presenta adecuadas evidencias de fiabilidad y validez. $\mathrm{La}$ fiabilidad de esta nueva versión es ligeramente menor a la obtenida en el estudio 1, posiblemente debido a la disminución de ítems, ya que la longitud de un test influye en su fiabilidad (Martínez et al., 2006). La versión final del instrumento se presenta en el Apéndice 1.

\section{Discusión}

El objetivo de este trabajo fue desarrollar un cuestionario de HS para el contexto académico, en formato online, dirigido a estudiantes secundarios chilenos de establecimientos educativos vulnerables. La primera versión del instrumento se construyó con una estructura de cinco dimensiones, en base a lo planteado por Del-Prette et al. (1998). Luego de los dos estudios realizados, esta fue modificada a un modelo más simple, de tres factores y 15 ítems, obteniéndose un instrumento con adecuadas propiedades psicométricas y breve tiempo de aplicación.

Pese a que existen instrumentos disponibles en Iberoamérica para medir HS (Caballo, 1993; Eceiza et al., 2008; Ipiña et al., 2011; MirandaZapata et al., 2014), este es el primer instrumento construido especialmente para estudiantes secundarios y orientado a las HS necesarias en la vida académica, que además cuenta con evidencias de validez basadas en su contenido, estructura y relación con un criterio externo.

El CHS-A puede constituir una herramienta para favorecer el logro académico y la permanencia en el sistema educativo, ya que permite realizar una descripción rápida de las habilidades sociales de los estudiantes, y con esto, identificar posibles necesidades de intervención.

El formato online también resulta un aporte relevante, pues facilita la automatización de los procesos de digitación, análisis e interpretación de los resultados, permitiendo la generación de informes automáticos personalizados para los estudiantes.

En términos teóricos, una de las principales modificaciones realizadas al modelo inicial fue la eliminación del factor Auto-exposición a desconocidos o a situaciones nuevas, que luego de los análisis realizados se fusionó con ítems del factor Conversación y desenvoltura social. Se le dio a esta nueva dimensión el nombre de Conversación, ya que los datos sugieren que la exposición a desconocidos o a situaciones nuevas pareciera ser más bien un contexto en el que se pueden expresar ciertas habilidades, como la capacidad de entablar conversaciones, en lugar de ser una habilidad en sí misma.

Por otro lado, dos ítems diseñados inicialmente para la dimensión Conversación se agruparon con los de Asertividad, sugiriendo que para los estudiantes las situaciones descritas suponen un riesgo mayor de respuesta negativa (hablar con un profesor y hacer disertaciones frente a compañeros).

Otra modificación importante a la estructura factorial fue la fusión, en un solo factor, de las dos dimensiones afectivas del cuestionario, Expresión de afecto positivo y Autocontrol de la agresividad en situaciones aversivas, al que denominamos Autorregulación. Estos resultados sugieren que los estudiantes que regulan adecuadamente sus emociones serían capaces tanto de controlar su agresividad como de expresar afecto hacia otros.

Las dimensiones de la versión final del CHSA son las siguientes:

1. Asertividad: habilidad para expresar opiniones, defender derechos, pedir algo o expresar desacuerdo, en forma respetuosa y no agresiva, en situaciones donde es posible obtener una respuesta negativa.

2. Conversación: habilidad para iniciar y mantener conversaciones tanto con conocidos como con desconocidos y relacionarse adecuadamente con otros en situaciones cotidianas. 
3. Autorregulación: habilidad para controlar las propias emociones y expresarlas adecuadamente. Implica tanto controlar las sensaciones desagradables para no reaccionar de forma agresiva, como expresar cariño y apoyo a otros cuando lo necesitan.

Esta estructura de tres factores tiene cierta similaridad con distintas conceptualizaciones del constructo de HS (Del-Prette et al., 1998; García, Cabanillas, Morán \& Olaz, 2014; Olaz et al., 2009). Sin embargo, la principal diferencia con otras estructuras está en el menor número de factores del CHS-A, ya que agrupa habilidades que tradicionalmente se han considerado independientes. Tal es el caso de Asertividad, por ejemplo, que agruparía habilidades de expresión de opiniones y hablar en público, presentes de forma separada en la EMES-M (Caballo, 1993). Una segunda diferencia con otras estructuras es que al acotarse el contexto, las dimensiones resultantes no se conforman en función de éste, excluyendo factores tales como "Interacción con familiares" o "Expresión de cariño hacia los padres", presentes en otros instrumentos.

La estructura del CHS-A es muy similar a la propuesta por Lazarus (1973), quien plantea que existirían cuatro habilidades sociales: la habilidad de decir "no", la de hacer solicitudes, la de expresar sentimientos positivos y la de conversación. En el caso del CHS-A las dos primeras se agruparían en el factor de Asertividad, mientras que la tercera incluye además la regulación de afectos negativos.

Dado que aún no existe acuerdo respecto al número ni al carácter de los factores que componen el constructo, y que una dificultad importante es la falta de parsimonia al sugerirse un número excesivo de factores (Caballo, 2000, citado en Olaz et al., 2009), el presente trabajo puede constituir un aporte a la discusión sobre la dimensionalidad de las HS.

En cuanto a las diferencias por sexo en las dimensiones Asertividad y Autorregulación, presentes en ambos estudios, estas concuerdan con resultados de otras investigaciones, que indican diferencias a favor de los varones en asertividad y expresión de sentimientos negativos, así como una mayor habilidad de las mujeres para expresar sentimientos positivos y reconocer errores (Caballo et al., 2014; García et al., 2014;
Olaz et al., 2009), lo cual estaría asociado a estereotipos que marcan las pautas y roles de género aceptados por cada sociedad. Sin embargo, para hacer comparaciones entre grupos no basta considerar solo la significancia estadística, sino que debe tenerse en cuenta también la magnitud de las diferencias (Cohen, 1994), y en este caso, éstas son bastante pequeñas, por lo que resulta necesario seguir estudiando la relación entre el sexo y las puntuaciones obtenidas en el CHS-A.

Respecto a las limitaciones del instrumento, si bien la fiabilidad resulta adecuada para usos descriptivos, es necesario revisarla para su uso con fines de orientación, ya que para la toma de decisiones a partir de los resultados de un instrumento se recomiendan valores más altos (Martínez et al., 2006; Prieto \& Delgado, 2010).

Asimismo, se observa una menor fiabilidad en la última versión, debido probablemente a la disminución de ítems en dos de sus dimensiones. Por ello se sugiere para futuras investigaciones la construcción de nuevos ítems que permitan hacer más robusta la medición, especialmente para la dimensión Autorregulación, que quedó con menos reactivos. Dado que también es la dimensión que presenta una mayor tendencia hacia puntuaciones altas se hace necesario volver a revisarla en próximos estudios, ya que podría estar siendo afectada por la deseabilidad social.

Son también tareas pendientes considerar evidencias de validez predictiva que permitan establecer una asociación entre los resultados en el CHS-A y la integración social de los estudiantes en sistema educativo, así como la evaluación del funcionamiento del instrumento en formato de lápiz y papel, para facilitar su uso en zonas con escaso acceso a internet.

\section{Referencias}

Bravo, A., \& Herrera, L. (2011). Convivencia escolar en Educación Primaria. Las habilidades sociales del alumnado como variable moduladora. DEDiCA Revista De EducaçãO e Humanidades, 1, 173-212. doi:10.30827/dreh.v0i1.7166

Abad, F. J., Olea, J., Ponsoda, V., \& García, C. (2011). Medición en ciencias sociales y de la salud. Madrid: Editorial Síntesis. 
Bandeira, M., Costa, M. N., Del Prette, Z. A., Del Prette, A., \& Gerk-Carneiro, E. (2000). Qualidades psicométricas do Inventário de Habilidades Sociais (IHS): Estudo sobre a estabilidade temporal e a validade concomitante. Estudos de Psicologia, 5(2), 401-419.

Bean, J. P., \& Eaton, S. (2001). The psychology underlying successful retention practices. Journal of College Student Retention Research. 3(1), 73-89.

Betina Lacunza, A., \& Contini de González, N. (2011). Las habilidades sociales en niños y adolescentes. Su importancia en la prevención de trastornos psicopatológicos. Fundamentos en Humanidades, 1(23), 159-182.

Bravo, A., \& Herrera, L. (2011). Convivencia escolar en Educación Primaria. Las habilidades sociales del alumnado como variable moduladora. DEDiCA Revista De EducaçãO e Humanidades, 1, 173-212. doi:10.30827/dreh.v0i1.7166

Caballo, V. (1983). Asertividad: Definiciones y dimensiones. Estudios de Psicología,4(13), 51-62. doi:10.1080/02109395.1983.10821343

Caballo, V. (1993). La multidimensionalidad conductual de las habilidades sociales: Propiedades psicométricas de una medida de autoinforme, la EMES-M. Psicología conductual, 1(2), 221-231.

Caballo, V. (2005). Manual de Evaluación y entrenamiento de las habilidades sociales. $\left(6^{\circ}\right.$ Edición). Madrid: Siglo XXI.

Caballo, V., Salazar, I., Olivares, P., Irurtia, M., Olivares, J., \& Toledo, R. (2014). Assessing social skills: The factorial structure and other psychometric properties of four self-report measures. Psicología Conductual, 22(3), 375399.

Caldarella, P., \& Merrell, K. (1997). Common dimensions of social skills of children and adolescents: A taxonomy of positive behaviors. School Psychology Review, 26(2), 264-278.

Canales, A., \& De los Ríos, D. (2007). Factores explicativos de la deserción universitaria. Revista Calidad en la Educación, 26(1), 171202.

Canales, A., \& De los Ríos, D. (2009). Entendiendo la permanencia de estudiantes vulnerables en el sistema universitario chileno. Revista Calidad en la Educación, 30, 1-38.

Cohen, J. (1994). The earth is round $(\mathrm{p}<.05)$. American Psychologist, 49(12), 997-1003. doi:10.1037/0003-066X.49.12.997

Cohen Imach, S., Esterkind de Chein, A., Betina Lacunza, A., Caballero, S., \& Martinenghi, C. (2010). Habilidades sociales y contexto sociocultural. Un estudio con adolescentes a través del BAS-3. Revista Iberoamericana de Diagnóstico y Evaluación - e Avaliação Psicológica, 1(29), 167-185.

Cohen, S., Sherrod, D., \& Clark, M. (1986). Social skills and the stress-protective role of social support. Journal of Personality and Social Psychology, 50(5), 963-973. doi:10.1037/0022-3514.50.5.963

Coronel, C. P., Levin, M., \& Mejail, S. (2011). Las habilidades sociales en adolescentes tempranos de diferentes contextos socioeconómicos. Electronic Journal of Research in Educational Psychology, 9(1), 241-262.

Del-Prette, Z., Del-Prette, A., Mendes, M., Bandeira, M., Rios-Saldaña, M., Oliveira, A., Gerk-Carneiro, E., Falcone, E., \& Villa, M. (2004). Habilidades sociais de estudantes de Psicologia: Um estudo multicêntrico. Psicologia: Reflexão $e$ Crítica, 17(3), 341-350. doi:10.1590/S0102-79722004000300007

Del-Prette, Z., Del-Prette, A., \& Mendes, M. (1998). Análise de um inventário de habilidades sociais (IHS) em uma amostra de universitários. Psicologia: Teoria e pesquisa, 14(3), 219-228.

Del Prette, Z. A. P., \& Del prette, A. (2013). Social skills inventory (SSI-Del-Prette): characteristics and studies in Brazil. Social anxiety disorders: From theory to practice, 49-62.

Eceiza, M., Arrieta, M., \& Goñi, A. (2008). Habilidades sociales y contextos de la conducta social. Revista de Psicodidáctica, 13(1), 11-26. doi:10.1387/RevPsicodidact.228

Elliot, S. N., \& Gresham, F. M. (1991). Social skills intervention guide. Practical strategies for social skills training. Circle Pines, MN: American Guidance Service. 
Elosua, P., \& Zumbo, B. (2008). Coeficientes de fiabilidad para escalas de respuesta categórica ordenada. Psicothema, 20(4), 896-901.

Ethington, C. (1990). A psychological model of student persistence. Research in Higher Education, 31(3), 279-293. doi: 10.1007/BF00992313

Ferrando, P. J., \& Anguiano-Carrasco, C. (2010). El análisis factorial como técnica de investigación en psicología. Papeles del Psicólogo, 31(1), 18-33.

Galassi, M. D., \& Galassi, J. P. (1978). Assertion: A critical review. Psycotherapy: Theory, Research and Practice, 15(1), 16-29. doi:10.1037/h0085834

García, M., Cabanillas, G., Morán, V., \& Olaz, F. (2014). Diferencias de género en habilidades sociales en estudiantes universitarios de Argentina. Anuario Electrónico de Estudios en Comunicación Social "Disertaciones", 7(2), 114-135. doi:10.12804\%2Fdisertaciones.v7i2.4622

Hunder, J. (1995). Enhancing social competente in young students. Austin, TX: Pro-ed.

Inderbitzen, H. M., \& Foster, S. L. (1992). The teenage inventory of social skills: Development, reliability, and validity. Psychological Assessment, 4(4), 451.

Inglés, C. J., Hidalgo, M. D., Méndez, F. X., \& Inderbitzen, H. M. (2003). The Teenage Inventory of Social Skills: Reliability and validity of the Spanish translation. Faculty Publications, Department of Psychology. 505-510.

Inglés, C., Méndez, F., Hidalgo, M., Rosa, A., \& Orgilés, M. (2003). Cuestionarios, inventarios y escalas de ansiedad social para adolescentes: Una revisión crítica. Revista de Psicopatología y Psicología Clínica, 8(1), 121. doi:10.5944/rppc.vol.8.num.1.2003.3939

Inzunza Melo, B., Ortiz Moreira, L., Pérez Villalobos, C., Torres Araneda, G., McColl Calvo, P., Meyer Kother, A., Matus Betancourt, O., Bastías Vega, N., \& Bustamante Durán, C. (2015). Estructura factorial y confiabilidad del Cuestionario de Satisfacción Académica en Estudiantes de Medicina Chilenos. Revista Iberoamericana de Diagnóstico y Evaluación - e Avaliação Psicológica, 2(40), 73-82.
Ipiña, M., Molina, L., \& Reyna, C. (2011). Propiedades psicométricas de la Escala MESSY (versión autoinforme) en niños argentinos. Revista de Psicología (PUCP), 29(2), 245-264.

Lazarus, A. (1973). On assertive behavior: A brief note. Behavior Therapy, 4(5), 697-699. doi:10.1016/S0005-7894(73)80161-3

Martínez, R., Hernández, M., \& Hernández, M. (2006). Psicometría. Madrid: Alianza.

Matson, J. L., Rotatori, A. F., \& Helsel, W. J. (1983). Development of a rating scale to measure social skills in children: The Matson Evaluation of social skills with youngsters (MESSY). Behaviour Research and therapy, 21(4), 335-340.

Miranda-Zapata, E., Riquelme-Mella, E., Cifuentes-Cid, H., \& Riquelme-Bravo, P. (2014). Análisis factorial confirmatorio de la Escala de habilidades sociales en universitarios chilenos. Revista Latinoamericana de Psicología, 46(2), 73-82. doi:10.1016/S0120-0534(14)70010-X

Muthén, B. O. (1998-2004). Mplus technical appendices. Los Angeles, CA: Muthén \& Muthén.

Newsom, J, (2005). Some clarifications and recommendations on fit indices http://web.pdx.edu/ newsomj/semclass/ho_fit .pdf

Olaz, F., Medrano, L., Greco, M., \& Del-Prette, Z. (2009). Argentinean adaptation of the social skills inventory IHS-Del-Prette. The Spanish Journal of Psychology, 12(02), 756-766. doi:10.1017/S1138741600002122

Organización para la Cooperación y el Desarrollo Económicos [OCDE]. (2013). Panorama de la educación 2013: Indicadores de la OCDE. Madrid: Santillana. doi:10.1787/eag-2013-es

OCDE. (2015). Panorama de la educación 2015: Indicadores de la OCDE. Madrid: Santillana. doi:10.1787/eag-2015-es

Pelechano, V. (1996). Habilidades interpersonales. Valencia: Promolibro

Pascarella, E., \& Chapman, D. (1983). A multiinstitutional, path analytic validation of Tinto's model of college withdrawal. American Educational Research Journal, 20(1), 87-102. 
Pascarella, E., \& Terenzini, P. (1980). Predicting freshman persistence and voluntary dropout decisions from a theoretical model. The Journal of Higher Education, 60-75. doi:10.2307/1981125

Piqueras, J., Olivares, J., Vera-Villarroel, P., Marzo, J., \& Kuhne, W. (2012). Invarianza factorial de la Escala para la detección de ansiedad social (EDAS) en adolescentes españoles y chilenos. Anales de Psicología, 28(1), 203-214.

Prieto, G., \& Delgado, A. (2010). Fiabilidad y validez. Papeles del Psicólogo, 31(1), 67-74.

Ramírez, S., \& Justicia, F. (2006). El maltrato entre escolares y otras conductas-problema para la convivencia. Revista Electrónica de Investigación Psicoeducativa, 4(2). 265-289.

Riggio, R. (1986). Assessment of basic social skills. Journal of Personality and Social Psychology, 51(3), 649. doi:10.1037/0022-3514.51.3.649

Riggio, R., Watring, K., \& Throckmorton, B. (1993). Social skills, social support, and psychosocial adjustment. Personality and Individual Differences, 15(3), 275-280. doi:10.1016/0191-8869(93)90217-Q

Robbins, S., Allen, J., Casillas, A., Peterson, C., \& Le, H. (2006). Unraveling the differential effects of motivational and skills, social, and self-management measures from traditional predictors of college outcomes. Journal of Educational Psychology, 98(3), 598-616. doi:10.1037/0022-0663.98.3.598

Robbins, S., Lauver, K., Le, H., Davis, D., Langley, R., \& Carlstrom, A. (2004). Do psychosocial and study skill factors predict college outcomes? A meta-analysis. Psychological Bulletin, 130(2), 261-288. doi:10.1037/0033-2909.130.2.261

Roca, E. (2005). Cómo mejorar tus habilidades sociales. Valencia: ACDE.

Spady, W. (1970). Dropouts from higher education: An interdisciplinary review and synthesis. Interchange, 19(1), 109-121.

Tinto, V. (1975). Dropout from Higher Education: A theoretical synthesis of recent research. Review of Educational Research, 45(1), 89125. doi:10.3102/00346543045001089
Tinto, V. (1993). Leaving college: Rethinking the cause and cures of student attrition (2nd ed.). Chicago: University of Chicago. doi:10.7208/chicago/9780226922461.001.0001

United Nations Educational, Scientific and Cultural Organisation, UNESCO (2012). Global Digest. Opportunities lost the impact of grade repetition and early school leaving. Montreal, Quebec: UNESCO Institute for Statistics. Obtenido en http://www.uis.unesco.org/Education/Docume nts/ged-2012-en.pdf

Vera-Villarroel, P., Rodríguez, J., Kuhne, W., Alcázar, A., Santibáñez, C., \& López-Pina, J. (2007). Propiedades psicométricas de la Escala para la Detección de la Ansiedad Social (EDAS) en una muestra de adolescentes chilenos. International Journal of Clinical and Health Psychology, 7(3), 795806.

Villar, E., Caparrós, B., Romero, S., \& Camps, L. (2009). Desarrollo del capital social en la universidad. Informe final del proyecto competitivo (EA2008-0241)-Programa Estudios y Análisis, del Ministerio de Educación.

Yang-Wallentin, F., Jöreskog, K. G., \& Luo, H. (2010). Confirmatory factor analysis of ordinal variables with misspecified models. Structural Equation Modeling, 17(3), 392-423. doi:10.1080/10705511.2010.489003 


\section{Apéndice 1}

\section{Versión Final del CHS-A}

$\mathrm{N}^{\mathrm{o}} \quad$ Ítem

2 Si alguien me interrumpe cuando estoy hablando, soy capaz de pedirle que me deje terminar.

3 Si hice sentir mal a un compañero le pido disculpas.

4 Aunque esté con personas conocidas, me cuesta integrarme a las conversaciones.

$5 \quad$ Si un profesor me llama la atención, reacciono de forma respetuosa.

6 Me cuesta terminar las conversaciones y en general me quedo esperando a que otros lo hagan.

$7 \quad$ Puedo hacer disertaciones frente a mis compañeros sin ponerme nervioso.

$8 \mathrm{Si}$ en un grupo de personas conocidas estoy en desacuerdo con la mayoría, puedo expresar mi opinión.

10 Evito acercarme a hablar con personas que parecen muy distintas a mí.

11 Si me interesa un tema de la clase, me acerco a conversar con el profesor.

12 Aunque necesite alguna información evito preguntar a desconocidos.

13 Si un amigo hace algo que me molesta, se lo hago saber.

14 Cuando un compañero se siente mal, trato de apoyarlo.

15 Cuando estoy con una persona que acabo de conocer, me cuesta mantener una conversación.

16 Logro tomar como bromas las "tallas" que me hacen mis compañeros y no enojarme por ellas.

17 Si un profesor dice algo con lo que no estoy de acuerdo, me atrevo a darle mi opinión.

Nota. Los números señalados corresponden a la versión de 17 ítems para facilitar la interpretación de los resultados y figuras. 